\title{
The simplified model of a magnetic body dropping in a pipe Xing Chen
}

\author{
School of North China Electric Power University, Baoding 071000, China; \\ hbdldxhgx@163.com
}

Keywords: magnetic body copper pipe magnetic force motion model

\begin{abstract}
It is a common phenomenon that when speaking of a magnetic drop from a copper pipe, we often ignore to study it carefully, just thinking that is too simple. In this paper, we try to establish a simplified model to represent the regular moving pattern of the magnetic body according to the law of electromagnetic induction, Ohm's law and Ampere's law. In the process, we find some elements affecting its movement, including magnetic field intensity, metal pipe's geometric size and its resistivity. Also, we find an interesting conclusion that the magnetic force exists a maximum. And it is an identified value determined by magnetic field intensity.
\end{abstract}

\section{Introduction}

It is a common scene about a magnetic body falling in a copper pipe [1], which our teacher often showed us to introduce electromagnetic induction. Whereas they did not interpret how it is effected. Actually, if we study it carefully, we would find they are not a simple case just as we have thought. First, we reaffirm the fact that you will find the magnetic body drop slower when you put it in a copper pipe compared with its drop freely. Then we explain something about the magnetic field distribution of the small body. And the magnetic field distribution can be presented as the figure followed.

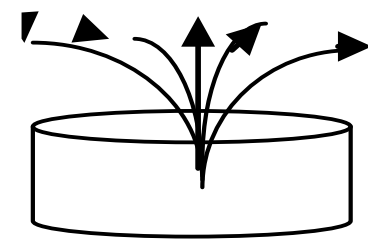

Fig.1 magnetic field distribution of a magnetic body ${ }^{[2]}$

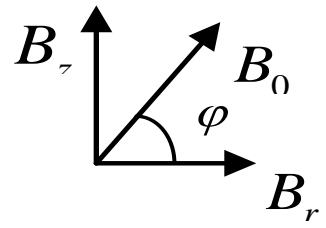

Fig.2 decomposition of magnetic induction line ${ }^{[3]}$

\section{Model building}

\subsection{Assumptions}

To make our analysis easier to understand, we do some assumptions in the model, and they are listed as followed.

(1) In this paper, aiming to deal with the model, we just take one end face of a small magnetic body into consideration when calculating the magnetic interact force.

(2) We ignore the magnetic flux leakage phenomenon when calculating the magnetic force.

(3) In the model we ignore air resistance in the process of falling.

\subsection{Section 1: The introduction of the experimental device}

In the experimental device, a small magnetic body is falling from a metal pipe (usually a copper pipe), whose diameter is slightly larger than the magnetic body. And this can be represented as the figure followed. When the small magnetic body's one end side coincides with the pipe, we drop it freely and start to time. And this time is the original time in our model. 


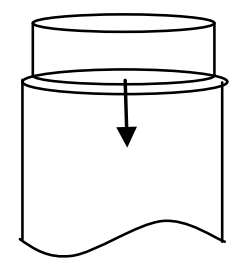

Fig. 3 the experimental device

\subsection{Section 2: The analysis of the resistance, emf and electric current}

In our model, we regard that the copper pipe is consisted of a series of coils and each of them is connected one by one to get a simplified model. Here we think that each coil's cross-sectional area is a unit area, and each coil's equivalent diameter is the average of inner and outer diameters, which means $r=r_{1}+r_{2}$. Then according to the Resistance Law[4]

$$
R=\rho \frac{l}{S}
$$

we get that the resistance of each coil is $R=\rho \pi\left(\mathrm{r}_{1}+r_{2}\right)$

Supposing that the small body's magnetic induction is $B_{0}$, the small magnetic body dropping. And what can change the magnetic flux is the axial component $B_{z}$. Consequently, each coil's magnetic flux is $B_{z} \bullet \pi\left(r_{1}+r_{2}\right)^{2} / 4$. Also we think that the small body experiences $d Z$ distance in $d t$ time, which also means it experiences $\mathrm{dZ}$ circles coil in $d t$ time. Referring to the Faraday's law of electromagnetic induction, the change of magnetic flux of coils is $\left(B_{r} \bullet \pi\left(r_{1}+r_{2}\right)^{2} / 4\right) \bullet \mathrm{dZ}$. Then we substitute them to the law of electromagnetic induction. As has indicated in the assumptions section, we don't take the magnetic flux leakage into consideration, then we can easily get that the induced emf is:

$$
\xi_{i}=-\frac{d \psi}{d t}=-\frac{\left(B_{r} \cdot\left(r_{1}+r_{2}\right) / 4\right) \cdot d Z}{\rho \cdot d t}
$$

Then the electric current of each coil can be worked out by the Ohm's law $I=\xi_{i} / R$. And it can be represented as :

$$
I=\xi_{i} / R=-\frac{\left(B_{z} \cdot\left(r_{1}+r_{2}\right) / 4\right) \cdot d Z}{\rho \cdot d t}
$$

\subsection{Section3: The analysis of magnetic force of each coil}

As the magnetic field is symmetrical (here we think the axis of magnetic body and metal pipe are overlapping), then the radial component $B_{z}$ can be regarded as a certain static value. And each coil's Ampere force can be represented as

$$
\vec{F}=\oint d \vec{F}=\oint B_{r} I d L=B_{r} I \bullet \pi\left(r_{1}+r_{2}\right)
$$

And we substitute (3) into (4), then the Ampere force is represented as

$$
F=\frac{B_{z} B_{r}}{4 \rho} \cdot \pi\left(\mathrm{r}_{1}+\mathrm{r}_{2}\right)^{2} \cdot \frac{d Z}{d t}
$$

In the vertical direction, we established its moving equations of motion according to Newton's Laws of Motion. While dropping it freely, it is affected by both the force of gravity and induced electric field force. In the vertical direction, we have

$$
M g-\frac{B_{z} B_{r}}{4 \rho} \cdot \pi\left(r_{1}+\mathrm{r}_{2}\right)^{2} \cdot \frac{d Z}{d t}=M \frac{d^{2} Z}{d t^{2}}
$$


Substitute (5) and $a=\frac{d^{2} Z}{d t^{2}}$ into (6) and we get

$$
M g-\frac{B_{z} B_{r}}{4 \rho} \cdot \pi\left(\mathrm{r}_{1}+\mathrm{r}_{2}\right)^{2} \cdot \frac{d Z}{d t}=M \frac{d^{2} Z}{d t^{2}}
$$

Performing integration of the equation and using the initial boundary conditions that $Z=0$ and $\frac{d Z}{d t}=0$ while $t=0$, then we get

$$
\frac{d Z}{d t}=g t-\frac{B_{z} B_{r}}{4 \rho M} \cdot \pi\left(\mathrm{r}_{1}+\mathrm{r}_{2}\right)^{2} Z
$$

Perform integration of (7) and its solution is

$$
Z=C e^{-\frac{B_{z} B_{r}}{4 \rho M} \pi\left(r_{1}+r_{2}\right)^{2} t}+\frac{4 \rho M g}{B_{z} B_{r} \pi\left(r_{1}+r_{2}\right)^{2}} t-\frac{16 \rho^{2} M^{2} g}{B_{z}^{2} B_{r}^{2} \pi^{2}\left(r_{1}+r_{2}\right)^{4}}
$$

Then we need to determine the value of $C$. Applying the boundary conditions $t=0, Z=0$ and we can calculate

$$
C=\frac{16 \rho^{2} M^{2} g}{B_{z}^{2} B_{r}^{2} \pi^{2}\left(r_{1}+r_{2}\right)^{4}}
$$

And equation (8) can be represented as

$$
Z=\frac{16 \rho^{2} M^{2} g}{B_{z}^{2} B_{r}^{2} \pi^{2}\left(\mathrm{r}_{1}+\mathrm{r}_{2}\right)^{4}}\left(e^{-\frac{B_{z} B_{r}}{4 \rho M} \cdot \pi\left(\mathrm{r}_{1}+\mathrm{r}_{2}\right)^{2} t}-1\right)+\frac{4 \rho M g}{B_{z} B_{r} \pi\left(\mathrm{r}_{1}+\mathrm{r}_{2}\right)^{2}} t
$$

Besides we perform derivation of (7), so its acceleration is

$$
a=\frac{d v}{d t}=g e^{-\frac{B_{2} B_{r}}{4 \rho M} \cdot \pi\left(r_{1}+r_{2}\right)^{2} t}
$$

And now we get its relationship of speed, acceleration ,and displacement with time.

\section{Further questions needed to be studied}

In our simplified model we just think one side's change of magnetic flux. What should be added if we consider both end sides in the process of falling?

What's the influence of wall thickness of pipe? In this paper we take their arithmetic average in the disposing of equivalent diameter, but would it better by using their logarithm average[4] ? And in the disposition of equivalent resistance we just think its cross-sectional area is a unit area, but should the area be related to the thickness of the pipe? And in the Calculus disposing process we get that the unit area is same with different pipe’s thickness. Maybe it’s amazing of Calculus[5].

\section{Summary}

In our simplified model we successfully get the equation of speed and acceleration of the small magnetic body when it is falling in the pipe, and we can find that there are many elements affecting the moving quality of the magnet, including the geometric dimensions $r_{1}, r_{2}$, the thickness and resistivity of the metal pipe. Among them we find that the geometric dimensions have huge importance on the moving. Here we need to indicate further discussion on the equation (5), $F=\frac{B_{z} B_{r}}{4 \rho} \cdot \pi\left(\mathrm{r}_{1}+\mathrm{r}_{2}\right)^{2} \cdot \frac{d Z}{d t}$. Actually, $B_{z} B_{r}$ existent a maximum because $B_{z}=B_{0} \cdot \sin \varphi$ and 
$B_{r}=B_{0} \cdot \cos \varphi$ and its maximum is $F=\frac{B_{0}^{2}}{8 \rho} \cdot \pi\left(\mathrm{r}_{1}+\mathrm{r}_{2}\right)^{2} \cdot \frac{d Z}{d t}$. Though this also changes with time, it exists a series of theoretical maximum.

\section{References}

[1]Sanhui Zhang. University Physics (third edition),Beijing, Tinghua university publisher,2008.

[2]Huiweng Guan. Movement analysis of a small magnet falling vertically brass. Laboratory Research and Exploration. 1995. 01,

[3] Pingping Gu . Interaction of the permanent magnet between moving conductor and a non-magnetic Hunan University. 2007.03.30

[4] Tongji University Department of Mathematics, Mathematics (6th Edition) Beijing: Higher Education Press; 2012.06. 